\title{
Análisis de la satisfacción y de la calidad percibida por las personas atendidas en los servicios sociales comunitarios del Centro Municipal de Servicios Sociales Delicias del Ayuntamiento de Zaragoza
}

\author{
Analysis of level of satisfaction and perceived quality of people attended \\ from Community Social Services of Municipal Social Services Center \\ Delicias of the City countil of Zaragoza
}

\author{
Eva DE LA PEÑA SALAS \\ Ayuntamiento de Zaragoza \\ eva_ps@yahoo.es
}

Recibido: $21 / 06 / 2013$

Revisado: $13 / 08 / 2013$

Aceptado: 08/11/2013

Disponible on line: $27 / 06 / 2014$

\begin{abstract}
Resumen
La calidad del servicio es un tema de actualidad presente en los discursos políticos, técnicos y de la sociedad en general, así como en los procesos de planificación, programas y proyectos sociales. Utilizando la escala SERVQUAL, conoceremos algunos aspectos de medida de la satisfacción y calidad percibida, a través de la mirada de las personas atendidas en un centro municipal de Servicios Sociales comunitarios, permitiéndonos, al equipo interdisciplinar, la mejor adaptación posible a las necesidades de estas personas. El ámbito más valorado es el de la «seguridad» y la «confianza» que transmiten los profesionales en el trato, mientras que el aspecto que presenta menor puntuación es su «capacidad de respuesta», entendida como la dificultad que tiene los profesionales de hacer frente y con rapidez a las demandas de los usuarios y usuarias. El índice general de satisfacción alcanza el 4.64 en una escala de 5, siendo mayoritariamente, el 76,4 por ciento, quienes valoran como «excelente» su sentimiento por los servicios que presta el centro municipal de Servicios Sociales de Delicias.
\end{abstract}

Palabras clave: calidad, calidad percibida, servicios sociales, SERVQUAL, modelos de evaluación.

\begin{abstract}
Service quality is a hot topic in the present political, technical and societal discourses in general, as well as in the planning processes, programs and projects. Using the SERVQUAL scale we will know some aspects of the measurements of satisfaction and perceived quality, through the eyes of the patients treated in a municipal community Social Services center. This will enable the interdisciplinary team the best possible adaptation to the needs of these people. The most valued aspect is the security and confidence of the staff, while the aspect presenting the lowest score is their problem-solving capacity, understood as the difficulty for professionals to deal with the demands of the users quickly. The general index of satisfaction reaches a 4.64 on a scale of 5 where $76.4 \%$ of the users considered «excellent» the feelings towards the services provided at Municipal Social Services Center of Delicias.
\end{abstract}

Keywords: quality, perceived quality, social services, SERVQUAL, evaluation models.

Referencia normalizada: De la Peña Salas, E. (2014): «Análisis de la satisfacción y de la calidad percibida por las personas atendidas en los servicios sociales comunitarios del Centro Municipal de Servicios Sociales Delicias del Ayuntamiento de Zaragoza». Cuadernos de Trabajo Social, 27(1); 115-125.

Sumario: Introducción. 1. Contexto de calidad. 2. Metodología del estudio. 3. Análisis de resultados. 4. Conclusiones. 5. Referencias bibliográficas. 


\section{Introducción}

La satisfacción de las personas con la atención recibida se concibe como una medida de control de calidad. En el presente artículo nos aproximaremos al concepto de calidad y percepción de la satisfacción del servicio en Servicios Sociales, y por otro lado, analizaremos los resultados de un estudio de investigación sobre la satisfacción de la atención percibida por los usuarios del Centro Municipal de Servicios Sociales Comunitarios de Delicias, del Ayuntamiento de Zaragoza.

La calidad del servicio es un tema de actualidad, sin embargo son pocos los estudios o experiencias llevadas a cabo para medir la satisfacción de la atención recibida por los usuarios de Servicios Sociales públicos. Además resulta complicado establecer comparaciones y agregar los resultados por utilizar metodologías, y enfoques dispares.

La orientación hacia la calidad para los profesionales de Servicios Sociales en general y del Trabajo Social como disciplina en particular debería constituirse como seña de identidad de nuestras intervenciones con los usuarios, más si cabe en la Comunidad Autónoma de Aragón, a través de la Ley5/2009 de Servicios Sociales de Aragón, donde se ha realizado una apuesta clara por la calidad de estos a nivel autonómico y municipal

\section{El contexto de la calidad}

\subsection{En busca de una definición de calidad}

La calidad no es un concepto nuevo. La preocupación por la calidad, por hacer bien las cosas, ha sido pauta de comportamiento de las sociedades en las diferentes etapas históricas, tal y como apunta Cánovas Sánchez (2002, p. 57).

En la aproximación del tema de calidad se pueden constatar dos cuestiones importantes. La primera de ellas es que no hay una definición homogénea sobre el concepto de calidad. Así lo afirma Medina Tornero (1999), indicando que el termino de calidad ha sido tan difundido que ha producido la transformación del significado por los diferentes matices, que puede dar lugar a sentidos múltiples o diversos. La segunda de ellas es la evidente evolución que ha sufrido el concepto de calidad. Se ha pasado de la mirada única en el producto/servicio, a centrarse en la mirada en los clientes y su satisfacción: la calidad total.

Veamos a continuación aquellas definiciones y/o perspectivas sobre calidad más afines para nuestra disciplina del Trabajo Social y de los Servicios Sociales. La etiología de la palabra «calidad» remite al vocablo latino qualitasatis. Nuestro diccionario de la Real Academia Española (2001) lo define como: «Propiedad o conjunto de propiedades inherentes a algo, que permiten juzgar su valor». Así mismo también lo define como «superioridad o excelencia». En el Diccionario de Trabajo Social, Fernández, Lorenzo y Vázquez (2012) definen calidad como: «el conjunto de características de un producto o servicio que cumplen con las expectativas del cliente para el cual fueron diseñados, satisfaciendo sus necesidades y expectativas»».

Se vinculan al enfoque integrador del concepto de calidad total que veíamos anteriormente, las características que apuntan Medina Tornero y Medina Ruiz (2011) acerca de sus principios son: mejora continua, satisfacción del cliente, compromiso de la dirección, adhesión del personal y eficiencia económica $\mathrm{y}$, desde esta óptica, concretamos que en este ámbito profesional nos interesa la mirada que concibe la calidad centrada en la satisfacción de los clientes.

\subsection{Calidad de servicio: la calidad en Servi- cios Sociales}

De la misma manera que no existe una definición universal de calidad, igualmente nos encontramos con dificultades para identificarla para los Servicios Sociales, como se indica en European Foundation for the improvement of living and working condicitions (2001):

No existe un concepto uniforme de calidad en los servicios de bienestar y existen diferencias entre los Estados miembros y entre los diferentes actores y grupos de interés (usuarios, trabajadores, directores, empleadores y administraciones estatales, regionales o locales) que están implicados en la mejora de la calidad (p. 55).

La calidad de servicio ha suscitado un especial interés, y del mismo modo que el concepto de calidad ha evolucionado, el de calidad de servicio también ha ido cambiando de una concepción más técnica y objetiva a otra más sub- 
jetiva y centrada en el usuario. Surge así, desde esta última concepción, el término de calidad percibida. Grönroos (1994) aportó la idea de que los usuarios comparaban sus expectativas con su percepción del servicio recibido, resultando de este proceso lo que se denomina la calidad percibida del usuario.

Bastantes autores han tratado de aclarar el concepto de calidad en Servicios Sociales. Veamos algunas de las definiciones más destacadas:

En el citado Diccionario de Trabajo Social, Fernández, Lorenzo y Vázquez defienden que la calidad integrada en éste implica mejorar la de la vida cotidiana y la laboral de los prestadores del servicio, potenciando la gestión de los mismos para que sean eficaces y eficientes y equilibrando los indicadores sociales, económicos y ambientales. La calidad de aquéllos depende en buena medida de los profesionales que trabajan de forma directa con los usuarios, quienes habrán que tener la capacidad de empatía, escucha, receptividad, motivación, apoyo emocional y observación, y la capacidad para elevar la autoestima.

La aportación que ya realiza sobre la calidad, como valor de la prestación de Servicios Sociales, Méndez De Valdivia (1999) establece que hay una serie de variables para medir la calidad y su nivel, entre las que destacan: el grado de accesibilidad del usuario a los Servicios Sociales, el nivel de coordinación entre instituciones, el tiempo de respuesta a la necesidad, el nivel de proximidad del usuario a los proyectos y programas sociales, así como el nivel de justificación del grado de satisfacción del usuario.

Según Gil Valenzuela y Escobar Rando (2004), la calidad en Servicios Sociales es un eje vertebrador de las intervenciones, igualando a los ciudadanos en las condiciones de acceso a los servicios, poniéndola en el centro de las intervenciones. Apuntan que la gestión de la calidad es en sí misma una estrategia de defensa ante las amenazas del sistema de Servicios Sociales, puesto que la monitorización de los procesos, la medición de los resultados, y la evaluación de la satisfacción de los usuarios son herramientas que pueden ayudar a la rentabilidad social del sistema, además de que sitúan las intervenciones y prácticas en los Servicios Sociales en un nivel científico similar al de los otros sistemas de protección.
Veamos a continuación lo más destacado de la calidad en los Servicios Sociales en tres niveles: internacional, nacional, y autonómico. En el primer nivel, uno de los trabajos pioneros en abordar las cuestiones de calidad en los Servicios Sociales europeos es la compilación de Evers, Haverinen, Leichsenring y Wistow (1997) donde se aborda el desafío que supone la introducción en los Servicios Sociales de conceptos sobre la garantía de calidad procedentes del ámbito empresarial. Se ofrece una panorámica de las experiencias desarrolladas en diversos países: Austria, Bélgica, Dinamarca, Finlandia, Alemania, Italia, Holanda, Noruega, Suecia y el Reino Unido.

A nivel nacional, en España el abordaje de los temas de calidad en el sistema de Servicios Sociales ha sido más reciente que en otros sistemas más consolidados, como el educativo o el sanitario. Como indica Giménez Bertomeu (2012) el interés por los asuntos de calidad ha ido lentamente en aumento, al igual que en el conjunto de la Administración Pública.

Sin duda a nivel nacional hay que mencionar, como un hito, lo que ha supuesto en relación a los criterios de calidad en los Servicios Sociales, la aprobación de la Ley 39/2006 de Promoción de la Autonomía Personal y Atención a las personas en situación de dependencia. Esta norma es la primera de rango nacional que regula aspectos relativos a la actuación de los Servicios Sociales, y dentro de ellos los de Atención Primaria, introduciendo criterios de calidad.

A nivel autonómico, la Ley 5/2009 de Servicios Sociales en Aragón ha implementado la apuesta firme y la incorporación de la calidad en materia de Servicios Sociales. El titulo VII se dedica exclusivamente a la calidad de los Servicios Sociales, resaltando el establecimiento de mecanismos de evaluación y garantía de sus criterios en todas las entidades prestadoras de Servicios Sociales, públicas y privadas.

\subsection{Modelos de evaluación de la calidad en la Administración Pública. Modelos de cali- dad en Servicios Sociales}

En este apartado me detendré en algunos de los modelos de evaluación de la calidad en la Administración Pública, principalmente en la Administración local, por la relación directa del 
estudio realizado en el marco del Ayuntamiento de Zaragoza, que más adelante veremos.

La Federación Española de Municipios y Provincias (2011) publica un interesante documento sobre Modelos de Evaluación para la Administración Local, donde se recopilan los de evaluación enfocados a la Administración local. Modelo de excelencia o de gestión de calidad europeo (Fundación Europea para la Gestión de Calidad EFQM.

- Modelo Iberoamericano de Excelencia en la Gestión.

- Modelos de la Organización Internacional para la Estandarización (ISO).

- Modelo de calidad para servicios contratados y concertados en Servicios Sociales con la empresa privada. AENOR (Asociación Española de Normalización y Certificación).

- Modelo de Marco Común de Evaluación (CAF).

- Modelo de Ciudadanía.

- Modelo Evaluación, Aprendizaje y Mejora (EVAM).

Tienen en común todos ellos el estar estructurados en torno a una serie de criterios que definen los aspectos a considerar para analizar tanto las actividades de la organización (liderazgo, planificación y estrategia, gestión de las personas, alianzas y recursos, y los procesos), como los resultados ( en los clientes, en las personas, en la sociedad y los resultados clave del rendimiento organizativo).

\subsection{Calidad percibida. Satisfacción del usuario en Servicios Sociales}

Se ha ido evolucionando desde el supuesto de que la satisfacción era el producto de una comparación, realizada por el sujeto entre sus expectativas y el resultado final obtenido, hacia la postura de que la satisfacción tiene también un componente afectivo, según afirma Cabrera Rodríguez Donoso Vázquez, Aneas Álvarez y Del Campo (2010).

La satisfacción ciudadana por la calidad de un servicio se relaciona con las características del mismo para atender necesidades de las personas a las que va destinado, pero también va unida a la percepción que el ciudadano tiene de cómo se presta y a la expectativa que tenía antes de recibir dicho servicio. Esta situación posicio- na al ciudadano como cliente de los mismos. Nos acogeremos para nuestro estudio al concepto de usuario/a entendiéndolo como el ciudadano/a que usa el servicio que le ofrece la Administración de forma gratuita, en la mayoría de las ocasiones, y en igualdad de condiciones.

Acerca de la participación de los usuarios en la determinación de los estándares de calidad de los servicios Domenech López y Giménez Bertomeu (2012) afirman que hay una escasez de experiencias de participación de los usuarios en la definición de la calidad de los Servicios Sociales.

Me detendré a continuación en la satisfacción del usuario de Servicios Sociales en la Comunidad Autónoma de Aragón y en el Ayuntamiento de Zaragoza, como referentes institucionales para el presente trabajo y estudio. En dicha Comunidad Autónoma tenemos que destacar la aprobación en del Plan de $\mathrm{Ca}$ lidad del Departamento de Sanidad, Bienestar Social y Familia (2013). Como vemos el Gobierno autonómico ha configurado un Plan específico en esta materia de calidad que coloca al usuario como protagonista en la toma de decisiones sobre su propio proceso, así como le reconoce el derecho a manifestar su opinión en el análisis de la satisfacción de la atención recibida. La misión de este Plan de Calidad consiste en proporcionar, a través de las organizaciones que conforman el Departamento, a los ciudadanos una «atención personal, adecuada, integral y segura, que garantice la accesibilidad y continuidad en la provisión de los servicios, respetando la autonomía de las personas y procurando satisfacer las necesidades y expectativas de la ciudadanía» (p. 3). Los objetivos y planes de acción se ordenan en relación a seis líneas estratégicas. Entre ellas se encuentra la línea estratégica tres de «orientación al usuario» que tiene relación directa con el conocimiento de la opinión y expectativas de los ciudadanos para introducir medidas de mejora.

Y en relación al Ayuntamiento de Zaragoza hay que destacar el Plan Director Estratégico de Servicios Sociales 2012/2020 de la Ciudad de Zaragoza (2013) por la importancia que concede a la satisfacción de los usuarios de Servicios Sociales. De reciente publicación, en febrero de 2013, el Área de Acción Social y Deportes de este consistorio, aprobó este Plan que establece, dentro de las cinco líneas estra- 
tégicas, la que se dedica exclusivamente a la cultura de la calidad, y en concreto establece una medida sobre la medición de la satisfacción de los usuarios.

\subsection{Estudios o experiencias en relación a la satisfacción de usuarios de Servicios Socia- les}

Como apuntaba en la introducción, no existen demasiados estudios sobre la satisfacción de los usuarios de Servicios Sociales comunitarios/primarios/básicos. Sí, es cierto que en relación a determinadas prestaciones sociales concretas o Servicios Sociales especializados, se han llevado a cabo más estudios sobre satisfacción. Además es difícil establecer comparaciones entre los estudios existentes sobre el tema puesto que los instrumentos o modelos para medir fueron diferentes, o se dieron en momentos de desarrollo de Servicios Sociales y en programas o servicios también diferentes. Se han llevado a cabo algunas experiencias de evaluación de la satisfacción de las personas usuarias $^{1}$ aunque con escasa trascendencia social.

\section{Metodología del estudio}

\subsection{Objeto del trabajo}

\section{Objetivo general:}

- Conocer la calidad de la atención a partir de la satisfacción percibida por los usuarios/as del CMSS de Delicias, del Ayuntamiento de Zaragoza como medida de calidad del servicio.

\section{Objetivos específicos:}

- Identificar las principales dimensiones de calidad según el instrumento SERVQUAL. Analizar y elaborar conclusiones.

\subsection{Población objetivo}

El presente estudio se va a realizar en el Centro Municipal de Servicios Sociales Comunitarios «El Ovalo» Delicias. Está ubicado en el barrio homónimo, uno de los más representativos por su extensión geográfica y por su densidad de población $(16,32$ por ciento del total de población de la ciudad vive en Delicias, con un total de 114.011 personas, con un índice de envejecimiento del 21,37 por ciento, y un 22,99 por ciento de población extranjera). Además es el Centro Municipal de Servicios Sociales Comunitarios, del total de los 27 centros que se distribuyen por la ciudad, que más nuevos expedientes ha abierto en el 2011 (19,7 por ciento del total de la ciudad) con un total de 1.486 expedientes nuevos, y contando actualmente con 14.621 expedientes activos.

La muestra del presente estudio comprende un total de 212 casos, que corresponden a los usuarios/as que tenían cita concertada con la trabajadora social o educador familiar/psicólogas en el Centro Municipal de Servicios Sociales Comunitarios durante la semana del 18 al 22 de Marzo de 2013.

\subsection{Instrumento de análisis}

Para medir la percepción de la satisfacción de la atención de los usuarios hemos utilizado la técnica del cuestionario que se basa en el mo-

${ }^{1}$ Sirvan de ejemplo la implantación del programa especifico de Mejora continua de la calidad en el Centro Municipal de Servicios Sociales Comunitarios de Alcázar de San Juan en Ciudad Real, de Moya Vaquero (2000); el proyecto «Indicadores de Calidad en los Servicios Sociales», presentado por Gimenez-Bertomeu y Domenech (2012) con la participación de 61 usuarios de tres Centros Municipales de Servicios Sociales Comunitarios a través de entrevistas en profundidad en la definición de los estándares de calidad; el Ayuntamiento de Madrid (2010) llevó a cabo estudio de satisfacción en Servicios Sociales especializados como ayuda a domicilio y también en Servicios Sociales Comunitarios; en el Ayuntamiento de Cuenca en Servicios Sociales, mediante el modelo de normativa ISO9001:2000, Del Pozo Herraiz, y Sánchez Pérez (2010) se implementó un modelo de gestión de la calidad; en el Ayuntamiento de Valdemoro, a través de la entidad INTRESS, Sánchez Aguado (2011) implementaron un sistema de mapa de procesos y de medición de satisfacción de los usuarios de Servicios Sociales; en el Servicio Social de base del Ayuntamiento de San Pedro del Pinatar junto con la Universidad de Murcia, García Pineda y otros ( 2010) realizaron un estudio para determinar el perfil de usuarios así como un estudio sobre satisfacción de estos; por su parte Medina Tornero. y Medina Ruiz. (2011) realizaron un estudio en dos Centros de Servicios Sociales del Ayuntamiento de Murcia sobre el análisis de la calidad percibida en usuarios de Servicios Sociales Comunitarios con un índice general de satisfacción utilizando la escala SERVQUAL de 6.21 sobre 7. 
delo centrado en la percepción subjetiva del usuario propuesto por Parasuraman, Zeithaml y Berry (1988), para medir variables tales como la tangibilidad, la fiabilidad, la capacidad de respuesta, la profesionalidad, la cortesía, la credibilidad, la seguridad y la accesibilidad. Se denomina SERVQUAL y se aplica para obtener indicadores sobre la calidad de servicio percibida por los usuarios.

El presente estudio solo ha medido la percepción del servicio o de la atención puesto que la mayoría de las personas encuestadas son usuarios habituales a estos servicios y poseían una confirmación (o no) de sus expectativas, por lo que no se podía medir el gap entre las expectativas y las percepciones del servicio prestado.

La escala utilizada para la medición del presente estudio es Likert de 5 puntos que van desde totalmente en desacuerdo (1) hasta totalmente de acuerdo (5). Se han formulado las preguntas de forma positiva por lo que no ha habido que invertir los valores.

Para el tratamiento y análisis de los datos se utilizo el paquete informático SPSS v.20 para Windows.

\section{Análisis de resultados}

\subsection{Datos sociodemográficos}

A continuación se presentan los datos sociodemográficos generales. Del total de personas hay que destacar que el 19,3 por ciento eran personas atendidas como primera cita con la trabajadora social frente al 80,7 por ciento que acudían a la entrevista con la trabajadora social o el/la educador familiar/psicóloga para cita de seguimiento.

El 53,3 por ciento eran mujeres frente al 46,7 por ciento de varones, por lo que podemos ver que, lejos del marcado predominio de aquélla como personas usuarias de Servicios Sociales que tradicionalmente se ha tenido, los porcentajes no demuestran una proporción muy desigual.

Mayoritariamente en relación al estado civil se encontraban casados o solteros con un 76,9 por ciento de la muestra, seguido de los separados y divorciados con un 13,7 por ciento.

La edad media de los participantes ha sido de 39,35 años con un mínimo de 18 años y un máximo de 84 años. Por segmentos de edad, el grupo más numeroso es el comprendido entre 31 y 40 años de edad (36,8 por ciento), seguido por el de 41 y 55 años de edad. Es importante destacar que el 91,5 por ciento tiene entre 18 y 55 años de edad.

En cuanto al país de procedencia el 30,2 por ciento es España y el 69,8 por ciento procede de un país extranjero, perteneciendo a uno de los hasta 29 países diferentes que se han registrado. En cuanto a la distribución de los grupos de origen extranjero, en primer lugar el más numeroso corresponde a Latinoamérica $(26,5$ por ciento), seguido de países norteafricanos como Marruecos, Argelia y Túnez (16,6 por ciento), países subsaharianos (14,1 por ciento), europeos como Rumania (10,8 por ciento) y el resto de países (1,9 por ciento).

En cuanto a la situación laboral de los encuestados observamos que el grupo mayoritario lo forman desempleados con un 74,1 por ciento de la muestra, le siguen las amas de casa (5,7 por ciento), los jubilados o pensionistas (3,8 por ciento), y otros o ns/nc ( 7,1 por ciento). Unicamente un 9,4 por ciento está en situación laboral activa, bien sea como trabajadores por cuenta ajena o como autónomos. El resultado nos indica una clara relación con las altas tasas de paro que predominan actualmente.

\subsection{Dimensiones de calidad percibida}

Una vez analizadas las variables sociodemográficas que conforman la muestra, procedemos a comentar los resultados que miden la calidad percibida, de acuerdo con las diferentes dimensiones de calidad que contempla el cuestionario utilizado, del servicio prestado desde el CMSS Delicias que ha sido objeto de estudio.

Como observamos en la Tabla 1 dentro de la dimensión de «elementos tangibles» podemos apreciar diferencias, siendo la percepción mejor valorada lo relacionado con el «aspecto y aseo de los profesionales» con un 4,81. Como ya se explicó en la metodología, la máxima puntuación de 5. Seguido en mejor percepción se encuentran las instalaciones del Centro $\mathrm{Mu}-$ nicipal de Servicios Sociales Comunitarios como «agradables y atractivas» $(4,58)$. Hay que destacar que el Centro Municipal de Servicios Sociales Comunitarios Delicias es un centro propio de Servicios Sociales, no compartido actualmente con otros servicios y relativamente nuevo, inaugurado en 2011. Sin embargo 


\begin{tabular}{|c|c|c|c|}
\hline & Media & Desv. tip. & Media \\
\hline $\begin{array}{l}\text { Las instalaciones del Centro de Servicios Sociales son agradables } \\
\text { y atractivas }\end{array}$ & 4,58 & 0,707 & \multirow{4}{*}{4,57} \\
\hline $\begin{array}{l}\text { Los profesionales del Centro de Servicios Sociales presentan buen } \\
\text { aspecto y van aseados }\end{array}$ & 4,81 & 0,459 & \\
\hline El Centro de Servicios Sociales cuenta con un equipamiento moderno & 4,55 & 0,711 & \\
\hline $\begin{array}{l}\text { Los materiales relacionados con el Centro (folletos explicativos, } \\
\text { cartelería) son visualmente atractivos }\end{array}$ & 4,33 & 0,769 & \\
\hline
\end{tabular}

Tabla 1. Resultado de la dimensión de calidad «elementos tangibles»

destaca que, en relación a la variable que mide si «el equipamiento es moderno» la puntuación baja hasta el 4,55. En relación a los materiales relacionados con el Centro, la percepción sobre si son «visualmente atractivos» es la más baja puntuación de esta dimensión. Indudablemente el usuario interactúa no solo con el profesional, sino también con aquellos elementos tangibles asociados al servicio.

En relación a la dimensión de «confianza» en la Tabla 2, la media es superior a los «elementos tangibles» con un 4,66. La variable que obtiene una mayor valoración es la referida a la «manera clara y comprensible de informar por parte de los profesionales del servicio», que obtiene un 4,75 , siendo además de la puntuación más alta de todo el cuestionario. Le siguen «el personal del centro realiza bien el trabajo desde la primera vez» $(4,70)$, «cumplir en el plazo prometido lo que se promete hacer» $(4,66)$, «la seguridad de las instalaciones» $(4,61)$ y en último lugar «mantener el sistema de información y registro al día» $(4,58)$.
La dimensión «capacidad de respuesta» en la Tabla 3 es la que obtiene la puntuación más baja de las cinco dimensiones con un 4,34 de media. La variable que mejor se valora es la del personal que «informa con precisión cuando concluirá el servicio ofrecido» $(4,56)$ que quizás pueda tener relación con la variable que veíamos en la dimensión de los profesionales del Centro de Servicios Sociales «me informan de una manera clara y comprensible», que era de las mejor puntuadas del cuestionario. Seguida está la variable sobre la «disponibilidad de los profesionales para atenderles»» $(4,47)$. Realizar sin embargo las tareas con «prontitud» $(4,35)$ y en concreto la variable de «atender rápidamente a las peticiones» $(3,99)$ es lo menos valorado. Además esta última variable es la puntuación mas baja de todo el cuestionario. Quizás esto tenga que ver con la demora en la cita programada con la que se atiende en este Centro Municipal de Servicios Sociales Comunitarios Delicias puesto que es de los que más volumen de atención tiene de

\begin{tabular}{|c|c|c|c|}
\hline & Media & Desv. tip. & Media \\
\hline $\begin{array}{l}\text { Cuando el personal del Centro de Servicios Sociales promete hacer } \\
\text { algo en cierto tiempo, lo cumple }\end{array}$ & 4,66 & 0,592 & \multirow{5}{*}{4,66} \\
\hline Las instalaciones del Centro de Servicios Sociales son seguras & 4,61 & 0,662 & \\
\hline $\begin{array}{l}\text { El personal del Centro de Servicios Sociales realiza bien su trabajo } \\
\text { desde la primera vez }\end{array}$ & 4,70 & 0,677 & \\
\hline $\begin{array}{l}\text { Los profesionales del Centro de Servicios Sociales me informan } \\
\text { de una manera clara y comprensible }\end{array}$ & 4,75 & 0,541 & \\
\hline $\begin{array}{l}\text { Los profesionales del Centro de Servicios Sociales mantienen } \\
\text { el sistema de información y registro al día }\end{array}$ & 4,58 & 0,630 & \\
\hline
\end{tabular}

Tabla 2. Resultados de la dimensión de calidad «confianza». 


\begin{tabular}{|c|c|c|c|}
\hline & Media & Desv. tip. & Media \\
\hline $\begin{array}{l}\text { El personal del Centro de Servicios Sociales no está demasiado } \\
\text { ocupado para responder rápidamente a mis peticiones }\end{array}$ & 3,99 & 1,097 & \multirow{4}{*}{4,34} \\
\hline $\begin{array}{l}\text { El personal del Centro de Servicios Sociales me informa } \\
\text { con precisión de cuando concluirá el servicio ofrecido }\end{array}$ & 4,56 & 0,690 & \\
\hline $\begin{array}{l}\text { Los profesionales del Centro de Servicios Sociales se encuentran } \\
\text { disponibles para atenderme }\end{array}$ & 4,47 & 0,915 & \\
\hline $\begin{array}{l}\text { Los trabajos relacionados con la acogida y seguimiento, tales como } \\
\text { entrevistas, informes, cuestionarios... se realizan con prontitud }\end{array}$ & 4,35 & 0,872 & \\
\hline
\end{tabular}

Tabla 3. Resultados de la dimensión de calidad «capacidad de respuesta»

\begin{tabular}{|c|c|c|c|}
\hline & Media & Desv. tip. & Media \\
\hline $\begin{array}{l}\text { El comportamiento de los profesionales del Centro de Servicios } \\
\text { Sociales me inspira confianza }\end{array}$ & 4,78 & 0,509 & \multirow{4}{*}{4,70} \\
\hline $\begin{array}{l}\text { El personal del Centro de Servicios Sociales está totalmente } \\
\text { cualificado para las tareas que realiza }\end{array}$ & 4,69 & 0,596 & \\
\hline $\begin{array}{l}\text { Los profesionales del Centro de Servicios Sociales se muestran } \\
\text { dispuestos a ayudarme, y su trato es considerado y amable }\end{array}$ & 4,77 & 0,547 & \\
\hline $\begin{array}{l}\text { Los profesionales del Centro de Servicios Sociales recogen } \\
\text { de forma adecuada mis quejas y sugerencias como usuario }\end{array}$ & 4,56 & 0,723 & \\
\hline
\end{tabular}

Tabla 4. Resultados de la dimensión de calidad «seguridad»

la ciudad, llegando incluso al mes de espera para dar una cita.

La dimensión de «seguridad», en la Tabla 4, es la mejor valorada de todas con una puntuación media del 4,70. La variable que mejor se puntúa es la «confianza que inspiran los profesionales del Centro» $(4,78)$ que además coincide que es la puntuación más alta junto con la que veíamos anteriormente del aspecto y aseo de los profesionales.

La dimensión «empatía», como refleja la Tabla 5, obtiene una puntuación media de 4,64. Las variables que miden la calidad percibida de esta dimensión tienen que ver con el «trato personalizado de los profesionales»), que es la puntuación más alta dentro de esta dimensión $(4,71)$, seguida de la «comprensión por parte de los profesionales de las necesidades como usuario» $(4,62)$. La satisfacción en relación a los «horarios del Centro» es la más baja puntuada en esta dimensión $(4,60)$.

Al final del cuestionario se incluyó un ítem de resumen para que los encuestados hicieran una valoración general sobre los sentimientos hacia los servicios que se prestan en el Centro Municipal de Servicios Sociales Comunitarios,

\begin{tabular}{|c|c|c|c|}
\hline & Media & Desv. tip. & Media \\
\hline $\begin{array}{l}\text { El personal del Centro de Servicios Sociales me ha dado un trato } \\
\text { personalizado }\end{array}$ & 4,71 & 0,645 & \multirow{3}{*}{4,64} \\
\hline $\begin{array}{l}\text { El Centro de Servicios Sociales tiene horarios que satisfacen } \\
\text { mis necesidades como usuario }\end{array}$ & 4,60 & 0,718 & \\
\hline $\begin{array}{l}\text { Los profesionales del Centro de Servicios Sociales comprenden } \\
\text { cuales son mis necesidades }\end{array}$ & 4,62 & 0,701 & \\
\hline
\end{tabular}

Tabla 5. Resultados de la dimensión de calidad «empatía» 


\begin{tabular}{|l|c|c|}
\hline & Media & Desv. tip. \\
\hline $\begin{array}{l}\text { Mis sentimientos hacia los servicios que presta el Centro de Servicios Sociales } \\
\text { pueden ser descritos como }(1=\text { muy insatisfactorios, } 5=\text { excelentes }\end{array}$ & $\mathbf{4 , 6 4}$ & 0,757 \\
\hline
\end{tabular}

Tabla 6. Resultados de «satisfacción general»

tal y como se observa en la Tabla 6 . Como vemos la puntuación media es de 4,64 siendo valorado como «excelente» por el 76,4 por ciento de la muestra.

Una vez analizado el resultado por variables de las dimensiones de calidad, se procede a relacionarlo con los factores sociodemográficos, con el fin de detectar posibles diferencias estadísticamente significativas. En la valoración media de las dimensiones según el sexo, estado civil, país de procedencia, tener hijos o no, nivel de formación, situación laboral, o el tipo de cita, no existen diferencias significativas entre quienes pertenecen a uno $\mathrm{u}$ a otro. Sin embargo, a la hora de analizar las variables por distintos grupos de edad encontramos diferencias significativas en la dimensión de «confianza» entre los grupos de edad de 18 a 30 años y el de 31 a 40 años. Yla diferencia estadísticamente significativa más evidente ha sido entre los usuarios que son atendidos por el Programa de Atención Familiar y el resto de la muestra en las dimensiones de «elementos tangibles», «capacidad de respuesta»y «satisfacción general». Además, es mayor y significativa la diferencia en la dimensión «empatía». Este Programa de Atención Familiar es de prevención familiar y la intervención del equipo, formado por trabajadora social, psicóloga y educador, es intensa en el tiempo, los objetivos y la proximidad a las familias.

\section{Conclusiones}

Los resultados desde un punto de vista global nos indican que no existen grandes diferencias entre las distintas dimensiones de calidad percibida que hemos medido. Si bien las tres dimensiones más altamente valoradas podríamos afirmar que están estrechamente vinculadas y que, a efectos de estudio, son importantes: «seguridad»,»confianza»y «empatía».

Las dimensiones que mejor han sido valoradas han sido las relacionadas con las capacidades del profesional del Centro Municipal de Servicios Sociales Comunitarios: en cuanto a la seguridad o conocimientos y cortesía de los empleados, así como la capacidad para transmitir seguridad y la capacidad para brindar el servicio de manera precisa y digna de confianza y por último la empatía, cuidado y atención individualizada. Las peor valoradas, por parte de los usuarios, han sido las ajenas a la labor del profesional, los elementos tangibles en cuanto a la apariencia de las instalaciones (materiales de comunicaciones, etc.) y la capacidad de respuesta que son aquellas variables que miden la rapidez con que se realiza el servicio entre otras.

La percepción de los usuarios/as sobre la labor profesional es de un 90,6 por ciento «satisfactoria»o «excelente» (14,2 por ciento y 70,6 por ciento respectivamente). Este resultado no es novedoso, si tenemos en cuenta que es un servicio de tipo asistencial, donde el factor humano prima por encima de las instalaciones. En un Centro Municipal de Servicios Sociales Comunitarios el contacto con el usuario es tan elevado como lo es la relación establecida entre el trabajador social (o profesional que le atiende psicólogo o educador familiar) y los usuarios atendidos.

Los centro municipales de Servicios Sociales Comunitarios del Ayuntamiento de Zaragoza en general, y el de Delicias en particular, están desbordados y los usuarios/as así lo han manifestado puesto que la pregunta que han puntuado más bajo ha sido la relacionada con que «los profesionales están demasiado ocupados para responder rápidamente a mis peticiones». Estos, debido al volumen de demanda, no siempre se encuentran disponibles para atender de forma rápida las demandas de los usuarios. Un horario de atención marcado por una agenda programada, con citas cada 20 minutos en el caso de los trabajadores sociales, hace que las citas se demoren y que los usuarios tengan que esperar, a veces, hasta un mes para ser atendidos por el trabajador social.

$\mathrm{Si}$ bien este estudio solo hace referencia a un Centro Municipal de Servicios Sociales Comunitarios, de los 27 que existen en la ciudad 
de Zaragoza, podríamos concluir que nos encontramos ante un reto importante en los Servicios Sociales del Ayuntamiento de la capital en lo relativo a la percepción de la calidad percibida por los usuarios de estos centros. Por un lado queremos cumplir con las líneas estratégicas marcadas, tanto en el Plan de Calidad del Departamento de Sanidad, Bienestar Social y Familia del Gobierno de Aragón (2013) como del Plan Director Estratégico de Servicios Sociales 2012-2020 de la Ciudad de Zaragoza (2013); y por otro lado y no menos importan- te, queremos realmente escuchar la voz de los usuarios, en relación a la percepción de cómo se sienten atendidos por los equipos de profesionales de los Servicios Sociales. Esto nos permitirá, sin lugar a dudas, establecer una mejor adaptación de nuestros servicios, implementar las modificaciones necesarias para dar respuesta a las personas con las que y para las que trabajamos día a día. Unas personas que, en el escenario socioeconómico actual, tienen mucho que opinar como ciudadanos y usuarios de un servicio público.

\section{Referencias bibliográficas}

Ayuntamiento de Madrid. (2010) Cuestionario de satisfacción de usuarios de los centros de Servicios Sociales. Madrid: Dirección General de Servicios Sociales y Atención a la Dependencia.

Barranco Expósito, C. (2002). La calidad percibida en Centros de Atención a Personas con Discapacidad Psíquica de Tenerife. Un enfoque integrado de la calidad de Vida, Calidad de Servicio y Calidad de Vida Laboral. Tenerife: Cabildo de Tenerife.

Barranco Expósito, C. (2006b). Ética, calidad integrada y burnout en las organizaciones de bienestar social: reflexiones y propuestas desde el Trabajo Social. Revista Servicios Sociales y Politica Social, 75, 105-120.

Cabrera Rodríguez, F.A., Donoso Vázquez, T., Aneas Álvarez, A. y Del Campo, J. (2010). Valoración de la satisfacción de usuarios de programas sociales: propuesta de un modelo de análisis. Revista de Educación, 351, 351-366.

Cánovas Sánchez, F. (2002). La cultura de la calidad desde una perspectiva histórica. Revista Documentación Social, 128, 57-80.

Del Pozo Herraiz, B. y Sánchez Pérez, M.C. (2010). Trabajando por la calidad desde los Servicios Sociales Básicos. Boletín informativo de Trabajo Social ,14. Disponible en: www.uclm.es/bits/sumario/79.asp (Consultado el 16 enero de 2013).

Domenech López Y. y Giménez Bertomeu, V.M. (2012). Percepciones sobre la calidad de los Servicios Sociales de las personas jóvenes usuarias: utilidades para el diseño de estándares de calidad. Revista de Estudios de Juventud, 97, 123-146.

European Commission. (2008). First Biennial Report on Social Services of general interest. Luxemburgo: Publications Office of the European Union. Disponible en http://eur-lex.europa.eu/LexUriServ/.do?uri=SEC:2008:2179:FIN:EN:PDF (Consultado el 24 de enero de 2013).

European Foundation for the Improvement of Living and Working Condicitions. (2001). Quality in social public services. Luxemburgo: Office for Official Publications of the European Communities.

EVERS, A.; HAVERINEN, R.; LEICHSENRING, K. AND WISTOW, G. (Eds.) (1997), Developing Quality in Personal Social Services: Concepts, Cases and Comments. Aldershot: Ashgate.

Federación Española de Municipios y Provincias. (2011). Modelos de Evaluación para la Administración Local. Madrid: FEMP. Disponible en: http://www.femp.es/files/566-979archivo/Modelos por ciento20de por ciento20Evaluaci por cientoC3 por cientoB3n por ciento20para por ciento20la por ciento20Administraci por cientoC3 por cientoB3n por ciento20Local por ciento20v2.pdf (consultado el 23 de enero de 2013).

Fernández, T., Lorenzo, R. y Vázquez, O. (eds.) (2012). Diccionario de Trabajo Social. Madrid: Alianza Editorial.

García Pineda M.L., Gago Cabrera, M.S., Gutiérrez Díez, L., Pini Gallego, F., García-Longoria, y Serrano M.P. (2010). Estudio del perfil de usuarios de un centro de Servicios Sociales. En A. Rodríguez Monge y E. Raya Lozano (coords.). Una Europa social y plural. Actas del VII Congreso estatal de Escuelas Universitarias de Trabajo Social. Granada: Universidad de Granada. 
Giménez Bertomeu, V.M. (2012). Investigación desde el Trabajo Social: la experiencia del proyecto INCASS sobre estándares de calidad en los Servicios Sociales. En O. Vázquez Aguado e Y. de la Fuente Robles (eds.) (2012). El Trabajo Social ante los desafios de un mundo en cambio (pp. 177-194). Huelva: Servicio de Publicaciones de la Universidad de Huelva.

Grönroos, C. (1994). Marketing y gestión de servicios. Madrid: Díaz de Santos.

Instituto para la Calidad de las ONG. (2011). Norma ONG Calidad, Versión 4². Madrid: ICONG. Disponible en: www.icong.org

Boletín Oficial de Aragón. (2009). Ley 5/2009, de 30 de Junio, de Servicios Sociales de Aragón Zaragoza: $B O A, 201$.

Likert, R. (1976). Una técnica para medir actitudes. En C.H.Wainerman (comp.), Escalas de medición en ciencias sociales (pp.199-260). Buenos Aires: Nueva Visión. .

IBM. (2011). Manual del usuario del sistema básico de IBM SPSS Statistics 20. IBM. Recuperado de: ftp://public.dhe.ibm.com/software/analytics/spss/documentation/statistics/20.0/es/ client/Manuals/IBM_SPSS_Statistics_Core_System_Users_Guide.pdf (Consultado el 14 de marzo de 2013).

Medina Tornero, M.E. (2000). Evaluación de la calidad asistencial del servicio de ayuda a domicilio. Murcia: Universidad de Murcia.

Medina Tornero, M.E. y Medina Ruiz, E. (2011).Análisis de la calidad percibida en usuarios/as de Servicios Sociales. Revista Zerbitzuan, 50, 85-100.

Medina Tornero, M.E., Medina Ruiz, E. y Vigueras Miralles, R. (2011). Calidad percibida en usuarios/as de Servicios Sociales Comunitarios. En III Congreso anual de la REPS: los actores de las políticas sociales en contextos de transformación.

Méndez de Valdivia, M. (1999). La calidad como factor clave en el desarrollo del sistema público de Servicios Sociales del año 2000. Cuadernos de Trabajo Social, 12, 79-102.

Moya Vaquero, E. (2000). Herramientas para gestionar la calidad de un Centro de Servicios Sociales. Revista de Servicios sociales y Política social, 49, 85-90.

Parasuraman, A., Zeithaml, V. y Berry, L. (1988). SERVQUAL: A multiple scale for measuring consumer perceptions of service quality. Journal of Retailing, 64, 12-40.

Gobierno de Aragón. (2013). Plan de Calidad del Departamento de Sanidad, Bienestar Social y Familia. Zaragoza: Dirección General de calidad y Atención al usuario,.

Ayuntamiento de Zaragoza. (2013).Plan Director Estratégico de Servicios Sociales 2012-2020. (2013). Zaragoza: Ârea de Acción Social y Deportes.

Real Academia Española. (2001). Diccionario de la Real Academia Española. 22a edición. Madrid: Real Academia Española.

Sánchez Aguado, A. (2011). La calidad en la gestión de los Servicios Sociales municipales: algunas experiencias de INTRESS. TS Nova. Trabajo Social y Servicios Sociales, 3, 49-54.

Social Protection Committee (2010). A voluntary European Quality Framework for Social Services. Recuperado de: http://ec.europa.eu/social//BlobServlet?docId=6140\&langId=en (Consultado el 24 de enero de 2013).

Zeithaml, V., Parasuraman, A. y Berry, L. (1993). Calidad total en la gestión de servicios: cómo lograr el equilibrio entre las percepciones y las expectativas de los consumidores. Madrid: Díaz de Santos. 